Hawton et al (Journal, May 1985, 146, 459-463) I feel sure that these authors would wish to acknowledge that a statistical association between suicidal behaviour and cruelty to children was demonstrated 13 years ago by McCulloch \& Philip (1972). Their important painstaking study deserves wider recognition than it has received hitherto.

King's College Hospital,

S. GREER London SES 9RS

Reference

MCCULlOCH, J. W. \& PHILIP, A. E. (1972) Suicidal Behaviour. Oxford: Pergamon.

\section{Research into Non-Organic Physical Symptoms} DEAR SiR,

Further psychiatric research is needed into nonorganic physical presentations and in this respect the study reported by Wilson-Barnett \& Trimble (Journal, June 1985, 146, 601-608) is welcome and interesting. There have been a number of similar studies lately in which psychiatric and psychological measures have been made in groups of patients with non-organic symptoms (for example, Bass et al, 1983; Blumer \& Heilbronn, 1982; Macdonald \& Bouchier, 1980; Gomez \& Dally, 1977; Beard et al, 1977). In most of these studies comparison has been made between a non-organic group and a group with diagnosed organic disease, and the nonorganic group has been found to have higher rates of psychiatric and psychological abnormalities. A conclusion common to most of the authors of these studies is that the physical symptoms represent unexpressed psychiatric disorder or unexpressed emotional distress.

In methodology all of these studies have limitations and the authors should perhaps have been more cautious in reaching their conclusions. All studies are cross-sectional in design and have generally investigated patients after they have been experiencing unexplained symptoms for several years. It therefore cannot be assumed that any abnormalities found are causal, whether these be in psychiatric health, personality inventories, history of childhood events, history of impaired sexual functioning, etc. That these abnormalities are effects of longstanding symptoms cannot be excluded. The organic comparison groups used in many of these studies have not been satisfactory. Severity of symptoms has never been matched, and it is possible that symptom severity has been less in the organic groups given that organic disease can often be treated and can sometimes remit. Furthermore, patients in these groups have known that an explanation for symptoms has been found and that treatment should follow.

Another criticism that can be made of the report by Wilson-Barnett \& Trimble and of the other studies referred to, is that the non-organic group was analysed as a single one, and conclusions appear to refer to all patients. This group of patients may be very heterogeneous and subclassification may be helpful. Patients in whom psychiatric disorder, masked or unmasked, seems to be the explanation for symptoms could be analysed separately. The remainder could be subclassified in terms of physical symptom variables seldom described in detail in the above studies-duration, course, nature of onset, severity in terms of distress and disability, history of frequent non-organic consultations, presence of illness fears, among others.

The sort of research needed to answer my points would be formidable, but until it is done I do not think we can claim to understand non-organic physical symptomatology with any certainty. DONALD I. MELVILLE

\section{Royal South Hants Hospital,}

Southampton SO9 $4 P E$

\section{Reterences}

BASS, C.. WADE, C., HAND, D. \& JACKSON, G. (1983) Patients with angina with normal and near normal coronary arteries: clinical and psychosocial state 12 months after angiogram. British Medical Jourrial, 207, 1505-1508.

BEARD, R. W., BELSEY, E. M., LIEBERMAN, B. A. \& WILKINSON, J. L. M. (1977) Pelvic pain in women. American Journal of Obstetrics and Gynaecology, 128, 566-570.

BLUMER, D. \& HEILBRONN, M. (1982) Chronic pain as a variant of depressive disease. The pain-prone disorder. Journal of Nervous and Mental Disease, 170, 381-394.

GOMEZ, J. \& DALLY, P. (1977) Psychologically mediated abdominal pain in surgical and medical out patient clinics. British Medical Journal, 1, 1451-1453.

MACDONALD, A. J. \& BOUCHIER, I. A. D. (1980) Non-organic gastrointestinal illness: a medical and psychiatric study. British Journal of Psychiatry, 136, 276-283.

\section{The PSE in Different Cultures}

DEAR SIR,

Swartz, Ben-Arie and Teggin (Journal, April 1985, 146, 391-394) provide a useful discussion of the rather exiguous opportunities in PSE9 for rating 'subcultural delusions or hallucinations'. I hope they will find the tenth edition, now under development, more satisfactory. The solution is indeed to provide local supplements and, if possible, also to translate these into English.

However, many apparently culture-specific 